log-binomial regression to assess the relationship between alcohol consumption patterns and sexual partnership concurrency.

Results Among 984 sexually active respondents, the median age was 23 years, with a majority having never married ( $N=705,72 \%$ ), received some high school education $(\mathrm{N}=546,55 \%)$, did not receive any income in past 3 months $(\mathrm{N}=621,63 \%)$, and had spent the past 7 nights at home $(\mathrm{N}=905,92 \%)$. Approximately $25 \%(\mathrm{~N}=123)$ of men reported at least one ongoing, overlapping relationship and $26 \%(\mathrm{~N}=131)$ had an AUDIT score $\geq 8$. Among women, $28(6 \%)$ reported concurrent sexual partners and only $6(1 \%)$ reported hazardous alcohol consumption patterns. For males, the prevalence ratio of hazardous alcohol consumption and sexual partner concurrency was 3.44 (95\% CI: 2.26, 5.23). Given the low prevalence of hazardous drinking among women we could not assess the relationship with sexual partner concurrency.

Conclusions Hazardous drinking among men was associated with sexual partner concurrency was associated with hazardous alcohol drinkers. Sexual risk reduction interventions and alcohol education are strongly needed for men in this setting.

\section{P4.069 PERCEIVED IMPORTANCE OF STI/HIV PARTNER NOTIFICATION AMONG MEN WHO HAVE SEX WITH MEN (MSM) AND TRANSGENDER WOMEN (TW) IN LIMA AND CALLAO, PERU (2010)}

doi:10.1136/sextrans-2013-051184.0967

1,2E Segura, ${ }^{2} \mathrm{~A}$ Perez-Brumer, ${ }^{3} \mathrm{~J}$ Sanchez, ${ }^{3} \mathrm{~J}$ Peinado, ${ }^{3} \mathrm{~J}$ Salvatierra, ${ }^{3} \mathrm{~J}$ Lama, ${ }^{2} \mathrm{~J}$ Clark. 'Universidad Peruana Cayetano Heredia, Lima, Peru; ' ${ }^{2}$ nniversity of California, Los Angeles. Program in Global Health, Lima, Peru; ${ }^{3}$ Asociacion Civil Impacta Salud y Educacion, Lima, Peru

Background In Peru, partner notification (PN) outcomes are correlated with its perceived importance. Data assessing perceptions of $\mathrm{PN}$ and its correlates among MSM/TW patient populations can help inform prevention interventions.

Methods MSM/TW in Lima diagnosed with HIV and/or STI within the last month were surveyed about their beliefs concerning both partner notification and practises among their peers. All analyses were stratified by partner type.

Results We recruited 390 MSM/TW (Ages: 18-60, Median: 28.5, IOR: 24-36) who identified as homosexual (63.0\%), bisexual (16.0\%), heterosexual (6.8\%), and TW (14.2\%). Overall, 93.3\% and $73.3 \%$ of respondents considered it "Very" or "Somewhat Important" to notify stable and casual partners, respectively. $73.5 \%$ of respondents believed that "Few" or "None" of their peers would notify their stable partners, while $84.7 \%$ believed the same for casual partners.

There was no association between perceived importance of notifying stable partners and beliefs about peer notification of stable partners. Among subjects who reported that "Few" or "None" of their peers would notify their casual partners, 31.7\% (52/164) of gays and $57.9 \%(22 / 38)$ of TW did not consider it important to notify casual partners while $90.9 \%$ (30/33) of gays and all (5/5) TW who reported "Most" or "All" peers would notify casual partners were likely to do the same themselves (all $p<0.05$ ). Low perceived importance of notification for any partner type was associated with identification as TW, low educational level, and "pasivo" sexual role (all $\mathrm{p}<0.05$ ) with consistently lower importance placed on notifying casual compared with stable partners.

Conclusions Perceptions about PN vary by partner type with casual partner notification considered least important. Perceived peer notification practises may reflect individual beliefs or social norms of notification behaviour, especially among TW and gayidentified MSM. Understanding individual-level, partner-level, factors and societal contexts that influence these perceptions will better inform interventions to promote PN.

\section{P4.070 THE GAP BETWEEN KNOWLEDGE OF HIV PREVENTION AND HIGH RISK INJECTION PRACTISE IN PEOPLE WHO INJECT DRUGS (PWID) IN BARNAUL, RUSSIA}

\author{
doi:10.1136/sextrans-2013-051184.0968
}

${ }^{1}$ E Demianenko, ${ }^{2} \mathbf{L}$ Zohrabyan, ${ }^{3} \mathrm{~L}$ Sultanov, ${ }^{1} \mathrm{~N}$ Vagaitseva, ${ }^{2} \mathrm{~J}$ Malkin, ${ }^{4} \mathrm{I}$ Toskin. ${ }^{\prime}$ Altay Kray Public Organization "AntiAIDS-Siberia", Barnaul, Russian Federation; ${ }^{2}$ UNAIDS, Moscow, Russian Federation; ${ }^{3}$ Center for AIDS and Infectious Disease Prevention of Altay Krai, Barnaul, Russian Federation; ${ }^{4}$ Department of Reproductive Health \& Research (RHR) World Health Organization, Geneva, Switzerland

Background HIV cases in PWID were increased by $8 \%$ in 2012. Prevention components such as VCT, skills building, condom and injecting equipment distribution were in place. Previous research suggests that greater HIV prevention knowledge does not necessarily translate into less risky behaviours. We investigated level of knowledge of HIV prevention and sharing paraphernalia in PWID.

Method 500 PWID surveyed in a cross-sectional survey (AugustOctober 2012). RDS sampling was used with 5 former PWID seeds. Socio-behavioural, knowledge and practise, HIV and HCV prevalence were calculated with RDSAT. Composite knowledge indicator was used to measure knowledge of main prevention measures and rejection of misconceptions about HIV transmission. PWID who responded to all questions correctly has been identified as having knowledge (PWID-K).

Results The sample was predominantly: male $(67.4 \%$, 95\% CI $62.3,72.7)$, 30-39 years old $(54.7 \%$, 95\% CI 49.1, 60.1), single (53.4\%, 95\% CI 48.0, 59.4), unemployed (67.3\%, 95\% 62.1, 72.5). Prevention coverage was $64.2 \%$ (95\% CI 58.3, 69.8). 59.9\% PWID (95\% CI 54.7, 65.2) correctly answered to knowledge questions. PWID-K injected $>2<9$ times a day in the last month $-78.7 \%$ (95\% CI 71.2, 87.1), without knowledge - 67.8\% (95\% CI 52.8, 84.8). The majority sample reported $(85.3 \%, 95 \%$ CI $80.9,92.1)$ sharing paraphernalia in the last month. PWID-K shared paraphernalia in $80.3 \%(95 \%$ CI 75.1, 90.2) and without knowledge $93.6 \%$ $(95 \%$ CI 86.8, 99.1). More than half of PWID-K injected in group of $1-5$ injectors $(59.6 \%, 95 \%$ CI $50.6,68.3)$, and $33.2 \%(95 \% \mathrm{CI}$ 25.3, 42.2) without knowledge. HIV prevalence in PWID-K was $34.5 \%(95 \%$ CI $27.6,41.5)$ and HCV $-74.0 \%$ (95\% CI 67.9, 80.3) with insignificant difference from PWID without knowledge.

Conclusions Inspire of high level of knowledge significant portion of PWID practise riskier behaviour which may explain high HIV/ HCV prevalence. Further research is vital to understand the reasons and develop effective intervention to stop transmission.

\section{P4.071 PREDICTORS OF CONDOM USE AMONGST HIV-INFECTED INDIVIDUALS RECEIVING ANTIRETROVIRAL THERAPY (ART)}

doi:10.1136/sextrans-2013-051184.0969

C Govender. Centre for the Alds Programme of Research in South Africa, Durban, South Africa

Background Influence of knowledge of HIV+ status on sexual behaviour has not been well studied in Africa, where positive prevention could potentially have the greatest impact.

Objective To determine the influence of HIV + status awareness and other social factors on condom use.

Method A behavioural questionnaire was administered to 642 patients in Durban, South Africa. Consistent condom use was defined as reported condom use during last sex act at months 6, 12, 18 and 24 of follow-up. Patients were divided into 2 groups: those who were aware of their HIV + status prior to study enrolment (Group A) and those who only learned their HIV+ status at the time of study enrolment (Group B). Generalized estimating equations were used to assess factors associated with condom use at last sex act over time. 